\title{
Hymen conservative techniques for vaginal surgery - a practical approach
}

\author{
Zaki Sleiman ${ }^{1}$, Amal Ayed ${ }^{2}$, Costas Christoforou ${ }^{3}$, Marco Noventa ${ }^{4}$, Amerigo Vitagliano ${ }^{4}$, \\ Antonio Simone Laganà ${ }^{5}$, Giovanni Buzzaccarini ${ }^{4}$
}

${ }^{1}$ Department of Obstetrics and Gynaecology, Lebanese American University, Beirut, Lebanon

2Department of Obstetrics and Gynaecology, Farwanya Hospital, MOH, Kuwait

${ }^{3}$ Christoforou Clinic, Cyprus

${ }^{4}$ Department of Women's and Children's Health, University of Padua, Italy

${ }^{5}$ Department of Obstetrics and Gynaecology, “Filippo Del Ponte” Hospital, University of Insubria, Varese, Italy

\begin{abstract}
Vaginal approach remains one of the safest and easiest surgical procedures to treat many gynaecological pathologies such as cervico-vaginal fibroids or vaginal septum.

These classical procedures are, however, hindered by the disruption of the integrity of the hymen, an issue that remains controversial if not unfavourable in certain conservative geographical areas such as the Middle East.

Herein, we describe 3 alternative surgical laparoscopic techniques to deal with the issue of hymen conservation. In the first 2 cases we suggest a laparoscopic approach through an anterior or a posterior colpotomy to treat large cervico-vaginal fibroids. In the third case, we propose a laparoscopic technique for the resection of a vaginal septum in a young girl with obstructed hemivagina and ipsilateral renal agenesis syndrome.
\end{abstract}

Key words: colpotomy, hymen preservation, vaginal fibroids, mini-laparotomy.

\section{Introduction}

Vaginal approaches remain among the safest and easiest methods to treat many gynaecological pathologies, including intrauterine, cervical, and vaginal lesions. They are also commonly adopted to evaluate many congenital anomalies associated with vaginal malformations. As such, vaginal surgery proves to be very helpful in addressing rare clinical scenarios such as transverse vaginal septum with hematocolpos, associated or not with other malformations including obstructed hemivagina and ipsilateral renal agenesis (OHVIRA) syndrome [1]. The major success of this procedure is, however, hindered by disruption of the integrity of the hymen [2], an issue that largely remains unfavourable and unacceptable among certain populations in conservative geographical areas such as the Middle East.

Vaginal leiomyomas or fibroids represent an uncommon entity with around 300 reported cases since the first description of this pathology by Denys de Leyden in 1733 [3]. The classical vaginal route is therefore commonly used to remove cervico-vaginal fibroids [4], thereby imposing a challenge in the Middle Eastern countries. The creation of alternative techniques to bypass the issue of hymen preservation is therefore of utmost importance to offer these patients the best surgical solutions while respecting cultural and religious backgrounds [5]. We herein report a case series of virgin patients treated for different vaginal pathologies by alternative surgical techniques that allow preservation of the hymenal ring.

\section{Cases}

\section{Case 1}

We published the first report of an alternative surgical technique in 2016 to treat a prolapsed pedunculated submucous leiomyoma that presented with symptomatic anaemia with a haemoglobin level of $8 \mathrm{~g} / \mathrm{dl}$ in a 20-year-old virgin patient [6].

The patient presented for a history of heavy menstrual bleeding of 6 months duration. She reported having regular cycles of 29-days duration and denied intermenstrual bleeding. She had had menarche when she was 11 years old and had a body mass index (BMI) of 21. She took no medications, and her family history was negative for bleeding disorders. Appropriate workup was done excluding anovulatory cycles, endocrine disorders such as thyroid dysfunction, hyperprolactinaemia, and polycystic ovary syndrome as well as bleeding disorders. However, the patient was found to be anaemic with a haemoglobin of $8 \mathrm{~g} / \mathrm{dl}$. 
On gynaecological inspection, the vulvar area appeared normal with an intact annular hymen. Abdominal examination was also unremarkable. Nevertheless, rectal examination revealed significant vaginal fullness. Pelvic transabdominal ultrasound subsequently showed a $5 \times 5 \mathrm{~cm}$ well-delineated solid hypo-echoic mass in the cervico-vaginal zone, filling the vaginal margins, clearly outside the endometrial cavity, while the uterus and adnexa appeared normal. Pelvic magnetic resonance imaging (MRI) confirmed the sonographic findings and suggested that this mass represented a prolapsed pedunculated submucous leiomyoma.

Transvaginal myomectomy was therefore discussed as a classical first-line surgical option. This approach was rejected by the patient and her family because of potential breach of the hymenal integrity during the procedure. Although an immediate post-myomectomy hymenal repair was proposed to the patient, she resorted to a laparoscopic abdominal approach.

Intra-operative inspection showed normal appearing uterus and adnexa. A bulky appearance located under the posterior vaginal wall was then noticed. As the introduction of a uterine manipulator was not approved by this virgin patient, we resorted to a different technique. As such, the uterus was sutured to the anterior pelvic wall in order to allow better exposure and sufficient access to the Douglas pouch. A vertical posterior colpotomy was performed and a firm $5 \times 5 \mathrm{~cm}$ regular pedunculated mass (Fig. 1) was identified and extracted through the incision by an electrical morcellator after cutting the peduncle and coagulation using bipolar electrocautery. The vaginal defect was repaired with absorbable Vicryl 0 laparoscopic simple sutures (Fig. 2). Histopathologic evaluation showed a benign leiomyoma with degenerating changes. The postoperative course was uneventful, and she was discharged home without any complications 24 hours after the surgery.

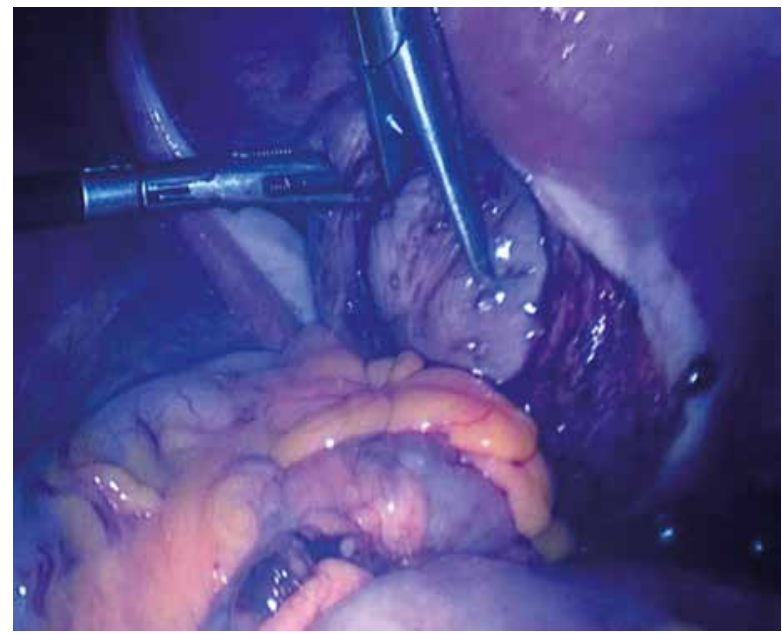

Fig. 1. After having performed the posterior colpotomy, the pedunculated fibroid was identified

\section{Cose 2}

In 2018, another young patient consulted for a similar presentation as in case 1 with a symptomatic vaginal fibroid of $4 \times 4 \mathrm{~cm}$.

The patient was a 22-year-old woman with a 1-year history of heavy menstrual bleeding. She had her menarche when she was 12 years old and reported regular cycles of 28-day duration. She denied intermenstrual bleeding or abnormal vaginal discharge. She did not take any medications and had a negative family history for bleeding disorders. Her BMI was 19. Examination revealed no thyroid enlargement, no signs of androgen excess, and no palpable abdominal or pelvic masses. Workup revealed anaemia with a haemoglobin of $9.6 \mathrm{~g} / \mathrm{dl}$. Her thyroid function tests, liver function tests, and prolactin were normal. She also had no evidence of bleeding disorders.

During the imaging investigation, the patient was found to have small bilateral endometriomas and a vaginal fibroid measuring $4 \times 4 \mathrm{~cm}$.

The same technique as above was proposed and accepted by the patient and her family to preserve hymenal integrity. Upon laparoscopic entry, we were surprised to find a frozen pelvis secondary to endometriosis. The Douglas pouch was completely filled with adhesions, preventing us from proceeding with the planned posterior colpotomy (Fig. 3). In light of such observations, we decided to dissect the bladder anteriorly, until we were able to identify the vaginal bulging secondary to mass effect. We subsequently performed an anterior vertical colpotomy instead of a posterior approach and extracted the fibroid through the peritoneal cavity (Fig. 4). The colpotomy was repaired with interrupted Vicryl 0 sutures.

\section{Case 3}

In 2019, we reported another alternative technique to treat a more complicated case of a symptomatic

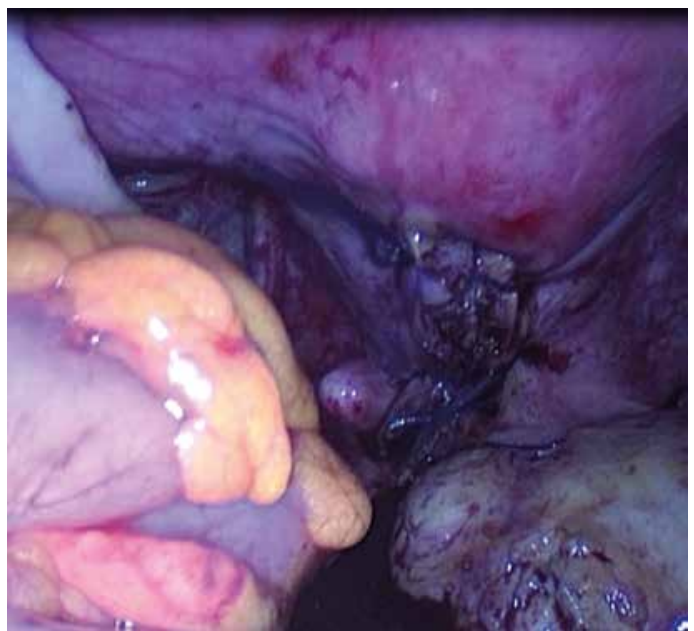

Fig. 2. The posterior colporrhaphy has been performed and the fibroid is yet to be extracted 


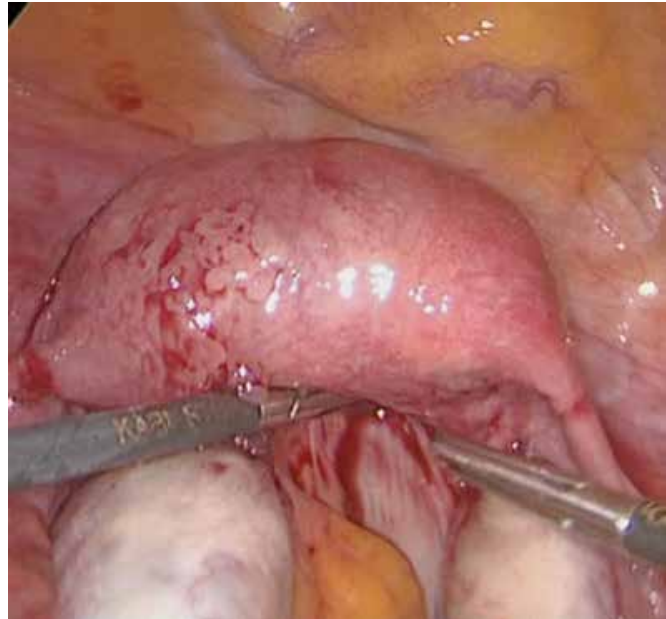

Fig. 3. The picture depicts the frozen pelvis and the difficult access for a posterior colpotomy

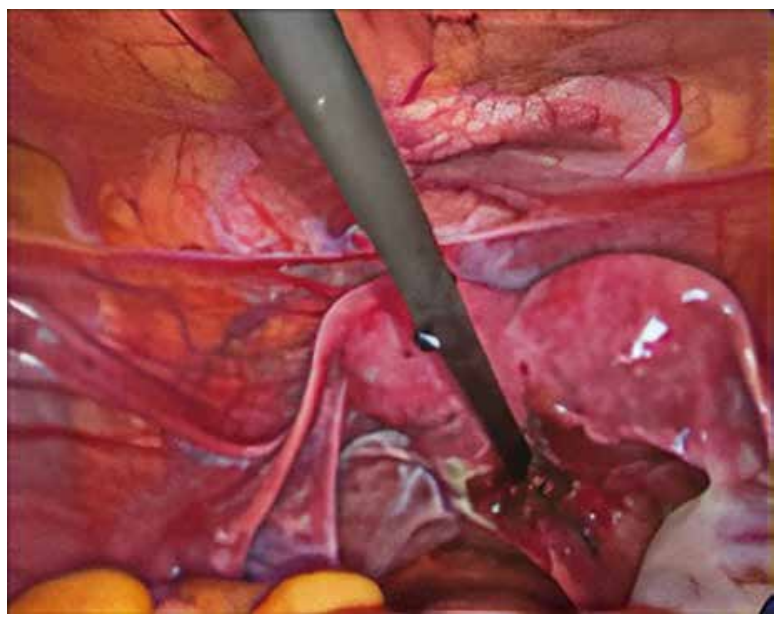

Fig. 5. The atraumatic graspers are introduced through the posterior colpotomy in a patient with obstructed hemivagina and ipsilateral renal agenesis syndrome

hematocolpos associated with OHVIRA syndrome in a 16-year-old virgin patient [7]. The patient was referred to our clinic for chronic pelvic pain and cyclic painful distension of the lower abdomen. Physical examination showed a young girl with normal stature and breast development, as well as axillary and pubic hair pattern. General inspection of the genital area also showed normal external genitalia. The patient and her family refused the speculum and the bimanual pelvic examination because it would have disrupted the hymenal integrity with subsequent loss of virginity. Instead, a digital rectal examination revealed a palpable soft mass occupying the vagina.

Workup was done and revealed normal FSH, TSH, and prolactin levels. A transabdominal ultrasound detected 2 uterine cavities, with fluid collection seen in the right one, a vaginal collection of $6 \times 4 \times 4 \mathrm{~cm}$, and the absence of the right kidney. A pelvic magnetic resonance imaging showed anatomical features compat-

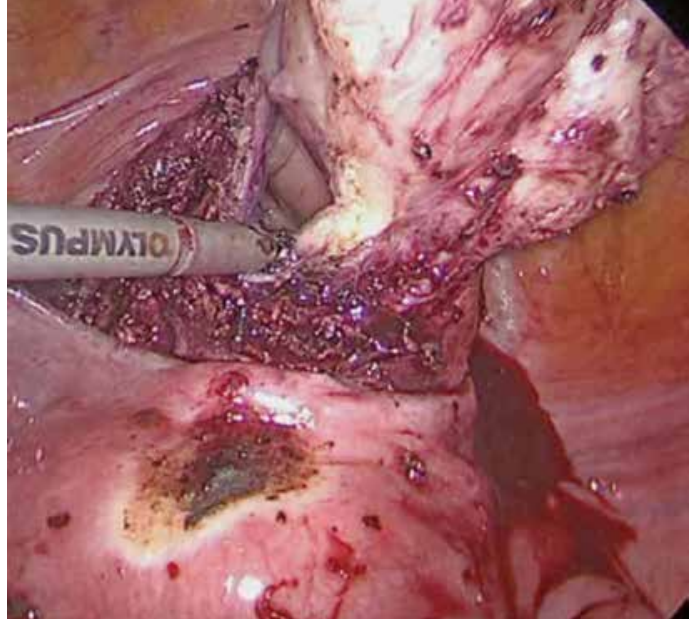

Fig. 4. The picture shows the anterior colpotomy and the extraction of the fibroid

ible with OHVIRA syndrome: a didelphic uterus along with a dilated right endometrial cavity contiguous with a right obstructed hemivagina. An exploratory laparoscopy and hysteroscopic resection of the septum through the vaginal route were offered to the patient, who was informed about the potential loss of hymenal integrity and about the possibility of hymenal repair after the procedure. She and her family radically refused the vaginal approach and consented to a laparoscopic assisted vaginal resection of the septum. The surgical inspection confirmed the presence of a didelphic uterus with a dilated right cavity along with normal adnexa and pelvic surfaces. A small posterior colpotomy was performed through which the obstructed hematocolpos was drained and washed. Fenestrated grasping atraumatic forceps were introduced to gently push the high vaginal septum downward towards the hymenal ring from the inside out, making it accessible for a classical resection/marsupialization without harming the hymen (Figs. 5, 6). The vaginal defect was repaired with absorbable laparoscopic simple sutures. The postoperative course was uneventful, and the patient was discharged without any complications 48 hours after the surgery.

\section{Discussion}

The preservation of an intact hymen has an undeniable social value in conservative areas such as the Middle East. For such societies, virginity portrayed by an intact hymen and is regarded as a symbol of sexual purity. Since some of the hymen types do not bleed during first intercourse, the inability to confirm virginity on the wedding night is therefore considered a major social concern and reflects serious social impacts on women [8]. Consequently, surgical interventions requiring the use of medical instruments through the vaginal route are largely rejected in those societies despite a low risk of harming the hymenal integrity. This imposes a limiting 

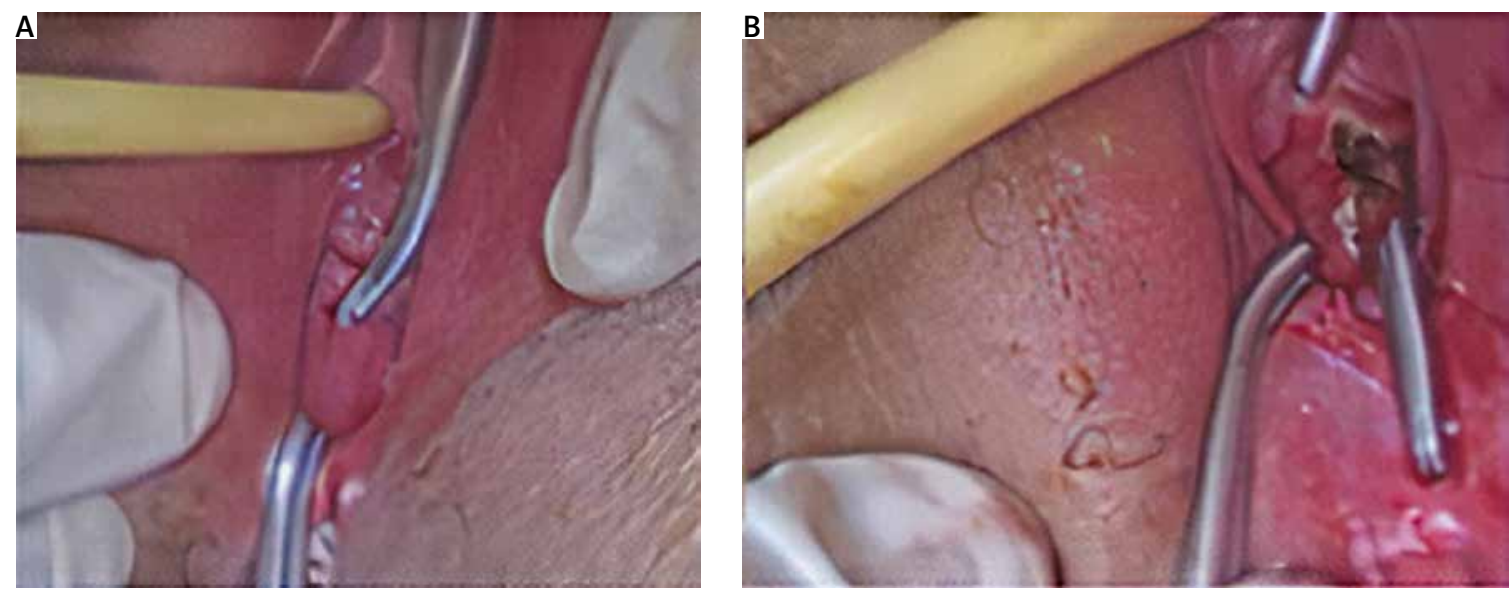

Fig. 6. Laparoscopic graspers are pushing the vaginal septum outside the hymenal ring (left side), followed by excision of the septum using the grasper as a landmark (right side)

and challenging barrier to gynaecologists trying to offer optimal approaches for the diagnosis and treatment of several illnesses. Alternative safer techniques that take into consideration the patient's desire should be adequately tailored upon the specific and peculiar case presented.

Vaginal myomectomy is the treatment of choice modality for prolapsed pedunculated submucous myomas [9]. To obtain optimal surgical outcomes with myomectomy, it is imperative to establish the extension and size of pedunculated submucosal leiomyomas into the myometrium before any intervention. This is best done by an intravaginal ultrasound [10], which could not have been performed in our virgin patients because of their desire to preserve the hymen. We resorted, instead, to transabdominal ultrasound and MRI to map the anatomy and establish a diagnosis, but the correct final diagnosis was only confirmed by visual inspection intraoperatively. Magnetic resonance imaging is also helpful in differentiating between fibroids and leiomyosarcomas or other vaginal malignancies before proceeding to surgical intervention. Classical vaginal myomectomy displays minimal associated morbidity in addition to breaching the hymenal integrity [11]. Possible techniques include hysteroscopic resection of the peduncle, non-hysteroscopic cutting or twisting of the peduncle, and morcellation or vaporization of myomatous nodules [12].

Hysteroscopy with vaginoscopic approach is a quick and well-tolerated alternative modality for the resection of vaginal fibroids, with few available reports establishing its feasibility in protecting hymenal integrity [9]. The hysteroscopic approach is, however, reserved for the resection of small pedunculated submucous myomas and has major limitations when operating on larger masses [13]. Mini-laparotomic anterior colpotomy was more recently reported as another hymenal integrity-sparing technique for the excision of a cervico-vaginal pedunculated submucous myoma in a virgin woman [14]. Nevertheless, in the presence of a skilled laparoscopic surgeon, a minimally invasive approach is always a safer alternative to laparotomy, with shorter hospital stay and better outcomes.

In our report of the first 2 patients, the myomas were large enough to preclude a vaginoscopic hymen-conserving approach. In the first case, we adopted a posterior colpotomy strategy because it provides easier access to the vaginal fibroid and avoids an unnecessary dissection of the bladder mandated by an anterior approach. Suturing the uterus to the abdominal wall is a key temporary step to facilitate anatomical exposure. In the second patient, the posterior access was, however, impossible because of endometriosis-related changes. Therefore, we decided to reproduce the laparotomic anterior approach by adopting a laparoscopic method. As such, the bladder was totally dissected in order to have enough access to perform the colpotomy, extract the fibroid, and perform the vaginal suturing. Both patients were discharged without complications 24 hours after the surgery.

Vaginal septum can be present as an isolated abnormality or be associated with other malformations such as those seen in the setting of OHVIRA syndrome. Vaginal access remains the 'gold standard' route in the management of vaginal septum. This approach is, however, hindered by different factors such as the septum's position, the presence of anatomic barriers, or the patient's concerns regarding her hymenal integrity. In our patient, who had a high septum and wished to conserve her virginity, we used laparoscopic assistance to push the high vaginal septum downward to the level of the hymenal ring and cut it at that level. We suggest that this method is also potentially useful in non-virgin women. In fact, when the septum is highly situated and the hematocolpos is not bulging enough, it is very difficult for the surgeon to initiate a vaginal incision, thereby risking an injury to the bladder anteriorly or to the rectum posteriorly. With the laparoscopic grasper 
introduced through a small posterior colpotomy and pushing the septum down, it is subsequently easier to identify anatomical landmarks and avoid injuries to the septum's nearby structures.

To continue with, new insights and consideration arise from peculiar but different cases. In particular, rare cases of vaginal atresia may require by some gynaecologists the vaginal vault intact. In these cases, colpotomy should be avoided [15]. Finally, attention should be given to the appropriate administration of new techniques. Although there is worldwide attention paid to minimally invasive surgery, such as vaginal natural orifice transluminal endoscopic surgery, with the aim to reduce post-operatory pain, blood losses, and aesthetic impairment, some approaches should be not considered in peculiar cases where the hymen integrity is necessary and undeniable $[16,17]$.

\section{Conclusions}

Respecting patients' social backgrounds is a crucial aspect of care delivered by an ethical healthcare professional. This, however, should not hinder the delivery of the best surgical treatments. Skilled surgeons are asked to use their knowledge and skills to become innovators, especially with the advent of novel sophisticated instrumentation. As such, preserving an intact hymenal ring is of utmost importance in certain traditional societies and may limit the feasibility of classical vaginal procedures. We suggest instead alternative laparoscopic techniques. All the above-described techniques are feasible, safe, and have favourable outcomes. They are, in addition, preferred by women who refuse transvaginal approaches. We encourage the adoption of these techniques in larger series in order to validate and reproduce the outcomes obtained.

\section{Disclosure}

The authors report no conflict of interest.

\section{References}

1. Matsuoka S, Kikuchi I, Kitade M, et al. Strategy for Laparoscopic cervical myomectomy. J Minim Invasive Gynecol 2010; 17:301-305.

2. Tug N, Sargın MA, Çelik A, Alp T, Yenidede I. Treatment of virgin OHVIRA Syndrome with haematometrocolpos by complete incision of vaginal septum without hymenotomy. J Clin Diagn Res 2015; 9: QD15-16.

3. Bennett HG, Ehrlich MM. Myoma of the vagina. Am J Obstet Gynecol 1941; 42: 314-320.

4. Young SB, Rose PG, Reuter KL. Vaginal fibromyomata: two cases with preoperative assessment, resection, and reconstruction. Obstet Gynecol 1991; 78: 972-974.

5. Awwad J, Nassar A, Usta I, Shaya M, Younes Z, Ghazeeri G. Attitudes of Lebanese university students towards surgical hymen reconstruction. Arch Sex Behav 2013; 42: 1627-1635.
6. Wehbe GS, Doughane M, Bitar R, Sleiman Z. Laparoscopic posterior Colpotomy for a Cervico-vaginal Leiomyoma: hymen conservative technique. Facts Views Vis Obgyn 2016; 8: 169-171.

7. Sleiman Z, Wehbe GS, Rassy EE, et al. A novel surgical intervention for an uncommon entity: laparoscopy-assisted resection of a vaginal septum in obstructed hemivagina and ipsilateral renal anomaly syndrome. J Laparoendosc Adv Surg Tech A 2019; 29: 714-716.

8. Brooks GG, Stage AH. The surgical management of prolapsed pedunculated submucous leiomyomas. Surg Gynecol Obstet 1975; 141: 397-398.

9. Golan A, Zachalka N, Lurie S, Sagiv R, Glezerman M. Vaginal removal of prolapsed pedunculated submucous myoma: A short, simple, and definitive procedure with minimal morbidity. Arch Gynecol Obstet 2005; 271: 11-13.

10. Cohen LS, Valle RF. Role of vaginal sonography and hysterosonography in the endoscopic treatment of uterine myomas. Fertil Steril 2000; 73: 197-204.

11. Kaivanara M. Virginity dilemma: re-creating virginity through hymenoplasty in Iran. Cult Heal Sex 2016; 18: 71-83.

12. Murakami T, Tamura M, Ozawa Y, Suzuki H, Terada Y, Okamura K. Safe techniques in surgery for hysteroscopic myomectomy. J Obstet Gynaecol Res; 2005; 31: 216-223.

13. Küçük T. When virginity does matter: rigid hysteroscopy for diagnostic and operative vaginoscopy-A series of 26 cases. J Minim Invasive Gynecol 2007; 14: 651-653.

14. Yalçin I, Pabuçcu E, Kahraman K, Sönmezer M. Mini-laparotomic colpotomy for a cervicovaginal leiomyoma: preservation of hymenal integrity. Int J Reprod Biomed 2016; 14: 217-220.

15. Naem A, Shamandi A, Al-Kurdy B. A rare form of Mayer-RokitanskyKüster-Hauser Syndrome associated with ovarian endometrioma: a case report. J Surg Case Rep 2020; 2020: rjaa393.

16. Buzzaccarini G, Stabile G, Török P, et al. Surgical approach for enlarged uteri: further tailoring of vNOTES hysterectomy. J Invest Surg 2021: 1-2.

17. Buzzaccarini G, Noventa M, d'Alterio MN, et al. vNOTES Hysterectomy: can it be considered the optimal approach for obese patients? J Invest Surg 2021; 1-2. 